\title{
INTRODUCCIÓN AL CONTENIDO DEL VOLUMEN
}

Este volumen contiene cuarenta trabajos académicos preparados $e x$ professo para el homenaje al profesor Wiaczesław Nowikow. Los artículos han sido distribuidos en seis secciones, atendiendo a su contenido temático, a fin de mostrar un orden coherente que, al mismo tiempo, descubra contenidos y aproximaciones comunes. Dado el campo de especialización del homenajeado, la mayoría de las contribuciones -treinta y una- pertenecen al ámbito de la lingüística, en tanto que otros nueve artículos integran la sección literaria, cuya presencia, no obstante, es fruto del interés filológico e hispanista del profesor Nowikow, más allá de lo estrictamente lingüístico.

La sección de "Estudios de lingüística" se ha dividido en cinco apartados titulados consecutivamente "Cuestiones de morfosintaxis", "Análisis léxico", "Enfoque pragmático", "Lengua en diacronía", "Lenguas y diversidad lingüística". La indudable riqueza de los planteamientos y temas presentes en la parte del volumen dedicada a la lingüística refleja, sin duda ninguna, los intereses de los autores, pero es también una prueba de que los numerosísimos estudios del profesor Nowikow han sido, directa o indirectamente, una fuente de inspiración para sus colaboradores, discípulos, colegas. Además, es de recalcar que el profesor Nowikow se ha acercado prácticamente a todos los campos que constituyen la parte lingüística del presente volumen.

El apartado dedicado a "Cuestiones de morfosintaxis" incluye nueve artículos: dos dedicados a la categoría gramatical del sustantivo, cinco en torno a cuestiones relativas a la categoría del verbo, y dos de análisis sintáctico. En el primer artículo, Margarita Lliteras tercia en la controversia del lenguaje inclusivo acerca de los nombres comunes en cuanto al género que no admiten cambio de desinencia, defendiendo que el carácter genérico del masculino es la propiedad distintiva de esta clase de nombres que designan clases de personas. En el siguiente 
artículo, Marta Wicherek describe el contenido semántico del morfema co- (junto con sus variantes con- y com-), demostrando que la agregación del prefijo co- a los vocablos puede afectar notablemente a su contenido semántico. A continuación, Juan de Dios Luque Durán y Lucía Luque Nadal, en un trabajo conjunto, superan la visión logicista que ha lastrado la comprensión y la explicación didáctica del uso de ser y estar en español a partir del reconocimiento de su polisemia y polifuncionalidad y de la existencia de múltiples y diferentes patrones que subyacen a la formación de enunciados con ser y con estar. Alexandre Veiga Rodríguez analiza la relación de simultaneidad en las formas del sistema temporal del verbo español a la luz de las caracterizaciones aspectuales de la última gramática académica, descubriendo empleos de dichas formas no caracterizables en términos aspectuales de imperfectividad. Los dos trabajos siguientes se hallan dedicados a los verbos de movimiento en español. Así, Nicole Delbecque pone el foco en el uso de la construcción reflexiva para denotar un movimiento direccional no causativo desde la perspectiva de la lingüística cognitiva, mostrando que la diferencia con la construcción no reflexiva afecta a la representación de la participación de la entidad sujeto. Por su parte, Cecylia Tatoj recurre a los conceptos de habitualidad y direccionalidad para presentar los equivalentes polacos del verbo ir, centrándose, además, en las dificultades que tienen los hispanohablantes a la hora de conceptualizar y emplear los verbos de movimiento polacos. A continuación, Luis Luque Toro aborda la cuestión de la recategorización del participio de pasado en su función sustantiva a causa de la influencia de la semántica, el conocimiento del mundo y la cognición en las unidades léxicas. Ya dentro de la sintaxis oracional, Tomás Jiménez Juliá analiza el valor paratáctico y el carácter completivo de que en los casos de porque o aunque, lo que determina diferencias gramaticales entre que y las conjunciones compuestas, y señala la identificación inadecuada del valor de una y otra forma. Finalmente, Janusz Pawlik estudia la variación del artículo en los grupos nominales GN modificados por una oración de relativo e intenta descubrir rasgos gramaticales de la oración relativa que decidan acerca de la referencia del conjunto nominal.

Las contribuciones dentro del bloque de "Análisis léxico" se concretan en seis estudios. En el de Marek Baran se contemplan las peculiaridades de las metáforas lexicalizadas propias del ámbito terminológico de la medicina, incluidos sus valores semánticos y pragmáticos y los epónimos y dobletes terminológicos. Los dos trabajos siguientes ponen el foco en la prensa deportiva. Así, Janusz Bień, mediante un análisis léxico-pragmático de titulares deportivos, examina las diferencias en cuanto a la valoración 
positiva de los éxitos deportivos del Real Madrid y del Fútbol Club Barcelona entre los medios digitales madrileños y catalanes. Antonio M. ${ }^{a}$ López González realiza un análisis léxico de las crónicas futbolísticas del diario Marca de partidos del Real Madrid y determina las designaciones de los conceptos principales del fútbol y la variación léxica y semántica en torno a estos conceptos. Pasando al léxico coloquial, Raúl Fernández Jódar presenta las bases para profundizar en la descripción del registro coloquial en español y polaco con vistas a realizar un análisis contrastivo de ambos conjuntos léxicos. Ya en el ámbito de la fraseología, Jerzy Szałek ahonda en la fraseología idiomática en busca de lo que se podría llamar acervo cultural hispánico, al tiempo que formula algunas tesis de una nueva rama fraseológica, que él llama idiosincrásica. El último trabajo de este bloque es el de Alicja Kacprzak, quien ofrece observaciones originales acerca de los neologismos eponímicos registrados en el discurso político francés, en torno a la figura de Emmanuel Macron dentro del contexto de la prensa diaria francesa.

La sección dedicada a estudios con un "Enfoque pragmático" cuenta con cinco trabajos. En el primero, Silvia Kaul de Marlangeon expone las convergencias entre los enfoques etológico-lingüístico y pragmáticosociocultural, dedicados a la indagación de las relaciones entre los usos lingüísticos y la respectiva cultura, sobre la base de las investigaciones acerca de las formas de tratamiento del mundo hispánico, tuteo, voseo y ustedeo. A continuación, Barbara Galant, después de repasar las distintas funciones del marcador hombre, trata de averiguar en qué medida estas últimas quedan reflejadas en las traducciones de esta partícula al polaco, para lo cual coteja secuencias comunicativas de novelas españolas contemporáneas con sus traducciones al polaco. Monika Lisowska estudia la superposición de los conceptos de 'cuantificación' e 'intensificación', causa del frecuente uso indiferenciado de ambos, prestando atención a los significados y funciones otorgados por la lingüística a esas nociones. En el siguiente artículo, Ewa Urbaniak demuestra que la reduplicación léxica desempeña un papel importante en la estructura argumentativa del discurso, siendo, además, uno de los recursos lingüísticos de la evaluación. El último trabajo de este bloque corresponde a un estudio conjunto de Agata Komorowska y Małgorzata Jędrusiak, en el que estas analizan desde el punto de vista pragmático algunas excepciones modales de uso del modo subjuntivo en las oraciones interrogativas subordinadas, cuyo modo habitual es el indicativo.

El apartado "Lengua en diacronía" viene compuesto de seis estudios. En el primero de ellos, Rafael Cano Aguilar lleva a cabo un análisis crítico de los estudios que se han ocupado de aspectos lingüísticos de la Primera 
crónica general (o Estoria de España alfonsí), de suma importancia para acercarse al español medieval. Seguidamente Emilio Montero Cartelle pone el foco en el Libro de los Exenplos por A.B.C., compuesto por estructuras discursivas medievales que recurren a las formas de expresión condicional de manera diferente en cada una de sus partes, lo que le permite estudiar la condicionalidad de manera individualizada. En el siguiente trabajo, Emilio Ridruejo, en el marco de los análisis de trasferencias entre lenguas de gran proximidad, somete a estudio el uso del participio de presente en aragonés medieval y en castellano, lo que le permite descubrir una mayor aceptación y generalización deeste fenómeno en el aragonés medieval. Tras este, Guillermo Rojo, en una aproximación a la historia de la lexicografía, analiza el tratamiento de los superlativos en -ísimo en el Diccionario de Autoridades, esto es, cuáles de estas formas figuraban en aquel diccionario y en qué medida. A continuación, el artículo de Witold Sobczak examina la cronología de la desaparición de las formas cantare y hubiere cantado del sistema temporal castellano, en sus variedades peninsular y americana, observando su empleo en las obras de la narrativa española de los siglos XVI-XIX recopiladas en el corpus CORDE. Cierra este bloque temático el estudio de Andrzej Zieliński, el cual se centra en el origen y evolución de las fórmulas de despedida formadas con el verbo de percepción ver y con el sustantivo vista.

El bloque titulado "Lenguas y diversidad lingüística" recoge artículos sobre la relación del español con otras lenguas a nivel internacional, peninsular, y dentro del mundo hispanohablante, así como artículos que estudian fenómenos lingüísticos desde el punto de vista de la lingüística comparada. Así, el artículo de Ángel López García-Molins discute el papel de la lengua y cultura españolas en el mundo, analizando sus relaciones e influencias lingüísticas, culturales y comerciales dentro de lo hispano y frente a lo hispanoamericano. Aneta Pawlak trata el tema de plurilingüismo de España, primero averiguando las diferencias entre los términos español y castellano y, después, presentando la complejidad del panorama lingüístico de España, en cuanto a sus lenguas cooficiales y sus modalidades lingüísticas. Ewa Kubiak, a partir de un estudio del paisaje lingüístico de la ciudad de Cusco, se fija en los quechuismos que aparecen en los nombres propios de los movimientos políticos de la región cusqueña y que sirven para subrayar la identidad indígena. Antonio Pamies Bertrán lleva a cabo un estudio de lingüística comparada acerca de las locuciones verbales, los verbos sintagmáticos separables, los verbos sintagmáticos inseparables y los verbos compuestos, incluidos los verbos con preposición y los verbos frasales. El último trabajo de esta sección, de Piotr Sorbet, analiza el fenómeno de la paragoge dentro de las 
lenguas románicas en el marco del cambio lingüístico para tratarlo como uno de sus mecanismos, y propone dos nuevas clasificaciones de sonidos paragógicos.

Las contribuciones que abordan diversas cuestiones literarias se han agrupado bajo el epígrafe "Estudios en torno al hecho literario", con el subtítulo de "Literatura, traducción y espectáculo", pues discurren por territorios propios de la historia de la lengua, la traducción, el teatro, la autobiografía, la poesía y la literatura comparada hispánica y polaca. El primer texto, de Beata Baczyńska y Monika Głowicka, traza la singular trayectoria de la voz bizarro, así como su presencia en fenómenos culturales actuales tales como los relatos de Olga Tokarczuk o la Bizarre fiction. La siguiente contribución, de Joaquín García-Medall, denuncia el peso de la tradición filológica hispánica que condena al lector de la literatura renacentista a la lectura de la muy arcaica traducción de Il libro del Cortegiano, llevada a cabo por Boscán hace casi cinco siglos. El enfoque traductológico se aplica asimismo en un texto de Anna Wendorff dedicado al teatro y, más concretamente, a la representación de Między nami dobrze jest de Dorota Masłowska en las tablas de un teatro madrileño. El mundo del teatro es explorado también en la contribución de Urszula Aszyk, que se propone repasar las representaciones actuales de La casa de Bernarda Alba, que confirman la universalidad del texto lorquiano. El estudio siguiente, obra de Amán Rosales Rodríguez, se centra en un tema de algún modo ya clásico -la construcción del yo literario- pero lo hace acercándose a lo autobiográfico desde la auto y metaficción. A caballo entre lo hispánico y lo polaco se sitúa la siguiente contribución, de Santiago Fortuño Llorens, que se adentra en los entresijos de la poesía de Wisława Szymborska, rastreando en sus poemas las alusiones a España y a su cultura. En cambio, el motivo del tango, analizado en el penúltimo capítulo, de Ewa Stala, brinda el tertium comparationis para relacionar las obras de la literatura argentina y polaca. A continuación, Ewa Kobyłecka-Piwońska y Agnieszka Kłosińska-Nachin, presentan un estudio de textos narrativos españoles y argentinos que, aunque de diversas maneras, conceptualizan el declive de los grandes relatos izquierdistas, concomitante con la representación de las respectivas transiciones políticas a la democracia. La sección de literatura se cierra con un trabajo de Mario García-Page, a partir de un corpus de poesía contemporánea española, sobre las figuras retóricas relacionadas con el ritmo y la rima con las que se crean juegos de palabras en la poesía. 